\title{
Thin-Film FTO-ZnO-CZTS Solar Cells Fully Deposited by Spray Pyrolysis
}

\author{
Salif Cisse $^{1,}$, , Maria Covei ${ }^{2}$, Jean Jude Domingo ${ }^{1}$, Anca Duta ${ }^{2}$ \\ ${ }^{1}$ Department of Physics, Faculty of Science and Technology, University of Cheikh Anta DIOP, Dakar, Senegal \\ ${ }^{2}$ Department of Renewable Energy and Recycling Systems, Faculty of Materials Science and Engineering, R\&D Institute of the Transilvania, \\ University of Brasov, Brasov, Romania
}

Email address:

slfcisse@gmail.com (S. Cisse), maria.covei@unitbv.ro (M. Covei), domingojean2001@yahoo.fr (J. J. Domingo), a.duta@unitbv.ro (A. Duta)

${ }^{*}$ Corresponding author

\section{To cite this article:}

Salif Cisse, Maria Covei, Jean Jude Domingo, Anca Duta. Thin-Film FTO-ZnO-CZTS Solar Cells Fully Deposited by Spray Pyrolysis. American Journal of Materials Synthesis and Processing. Vol. 4, No. 1, 2019, pp. 1-8. doi: 10.11648/j.ajmsp.20190401.11

Received: May 26, 2019; Accepted: June 18, 2019; Published: June 27, 2019

\begin{abstract}
Thin films of $\mathrm{ZnO}$, CZTS $\left(\mathrm{Cu}_{2} \mathrm{ZnSnS}_{4}\right)$ and $\mathrm{ZnO}-\mathrm{CZTS}$ were prepared by spray pyrolysis deposition (SPD). After spraying a precursor solution of $\mathrm{ZnO}$ directly onto fluorine-doped tin oxide (FTO) substrats, CZTS thin layers were sprayed onto layers of $\mathrm{ZnO}$ to forme a p-n junction. Some CZTS layers were directly spray onto fluorine-doped tin oxide substrats. $\mathrm{ZnO}$ is a wide band gap n-type material, consisting of abundant and nontoxic elements, and is thus expected to be a good substitute for CdS buffer layer in solar cells. In this paper, we report the study of CZTS and ZnO thin films synthesized by chemical spray pyrolysis (CSP) method. During the deposition of the ZnO thin films on the FTO substrats the number of sequences was varied from 20 to 40. The influence of the $\mathrm{ZnO}$ on the structural, optical, morphological and electrical properties of CZTS films was studied using various techniques. The X-ray diffraction studies showed the formation of kesterite $\left(\mathrm{Cu}_{2} \mathrm{ZnSnS}_{4}\right)$ phases with the peaks corresponding to (112), (220) and (312) planes. SEM study revealed a lack of uniformity of the surface of the CZTS layers sprayed onto the ZnO layers for a lower thickness of ZnO (FTO-ZnO20-CZTS). The band gap values of FTO-CZTS and FTO-ZnO-CZTS thin films were measured and found in the range $2.2-2.25 \mathrm{eV}$ which are in good agreement with the results reported $(2-2.2 \mathrm{eV})$. The morphological studies revealed the formation of some clusters randomly distributed on the film surface in the ZnO-containing layers, i.e. the FTO-ZnO20-CZTS and Finally, current-voltage measurements for different PV cells with maximum efficiency were carried out. A conversion efficiency ( $\eta$ ) of 5.99\% with fill factor $(\mathrm{FF})=18.8 \%$, open circuit voltage $(\mathrm{Voc})=0.54 \mathrm{~V}$, short circuit current density $(\mathrm{Jsc})=59 \mathrm{~mA} . \mathrm{cm} 2$ were recorded through the FTO-ZnO40-CZTS thin film PV cell.
\end{abstract}

Keywords: Spray Pyrolysis Deposition, Thin Films, $\mathrm{Cu}_{2} \mathrm{ZnSnS}_{4}$, Zinc Oxide

\section{Introduction}

One of the proposed ways for reducing the cost per watt is to reduce the manufacturing cost of the photovoltaic cell, thus to considerably reduce the amount of semiconductor materials in the composition of the cell and increase its lifetime. But also, since $\mathrm{Cd}$ is a highly toxic element, an alternative material is needed for the buffer layer to get a more environmental friendly device. In order to overcome these problems, several types of ternary and quaternary semiconductors can be proposed in the form of thin layers.
Among the most promising ternary semiconductors for the manufacture of thin-film solar cells are copper indium gallium diselenide $\mathrm{CuInGaSe}_{2}$ denoted CIGSe, in its chalcopyrite form. However, the production of CdTe and CIGSe based PV devices is expensive. Note also the toxicity of selenium (Se) and scarcity of indium (In) and gallium (Ga) [1].

$\mathrm{Cu} 2 \mathrm{ZnSnS} 4$ (CZTS) is one of the potential absorber materials for thin film heterojunction solar cells with ideal solar cell characteristics such as optimum band gap (1.4-1.5 eV) [2], high absorption coefficient $\left(10^{4} \mathrm{~cm}^{-1}\right)$ and p-type conductivity [2]. More, the availability of its elements which 
are relatively abundant and nontoxic in nature maks CZTS as one of the best candidate for PV technology [3]. Basically CZTS is derived from CIGSe by replacing In with $\mathrm{Zn}, \mathrm{Ga}$ with Sn, and Se with S [4]. Theoretical calculations have shown that conversion efficiency as high as $32.2 \%$ [5] is possible for CZTS thin film solar cells with a CZTS layer of few micrometers. A literature review indicates that several non-ideal recombination channels pose the main problem: (i) recombination at the interface between the kesterite and the CdS buffer. This is very likely due to an unfavourable clifflike band alignment between the absorber and the buffer. However, for pure selenide absorbers, this recombination path is not dominating, which could be due to a spike-like band alignment at the absorber-buffer interface. (ii) A second major recombination becomes obvious in a photoluminescence maximum well below the band gap, even in record efficiency absorbers. This is either due to a very high density of defects, comparable to the density of states in the band, or to stannite inclusions.

Several deposition methods such as magnetron sputtering [6], thermal evaporation [7], sol gel [8], screen printing [9], electrodeposition [10], photo-chemical deposition [11] and spray pyrolysis [12-13] are reported to grow CZTS thin films. However, spray pyrolysis deposition (SPD) has been identified as a low cost and versatile technique which could reduce cost per watt peak of PV modules [14]. In particular, SPD has been shown as non-expensive, non-vacuum and versatile technique for thin film growth. The highest efficiencies achieved in CZTS solar cell are still far below those reported for CdTe and CIGS technology. Up to date, the reported CZTS solar cell efficiencies are lower than $10 \%$ [15], because the control of secondary phases formation in CZTS represents a critical issue to obtain high efficiency devices [16]. However, the conversion efficiencies of $\mathrm{ZnO}-$ CZTS cells have exceeded $5 \%$ but are still lower than those of the best CdS-CZTS cells [17]. In order to achieve this, we investigated the structural and optical properties of CZTS, $\mathrm{ZnO}$ thin films synthesized by SPD. The aim of this work is to study the impact of a $\mathrm{ZnO}$ window layer on a FTO-ZnOCZTS PV cell by varying the number of $\mathrm{ZnO}$ spraying sequences. Since the $\mathrm{ZnO}$ layer plays a key role in thin-film solar cells, because it is a wide band gap oxide semiconductor with a direct energy gap of about $3.37 \mathrm{eV}$. As a consequence, $\mathrm{ZnO}$ absorbs $\mathrm{UV}$ radiation due to band-toband transitions, while it can be used as transparent conductive oxide (TCO) thin films, mainly for applications such as solar cells [18-19]. Also, ZnO (n-type semiconductor) is usually used as a window layer [20] and as a buffer layer for photovoltaic cells [21]. Further more, the $\mathrm{ZnO}$ thin film has to be highly transparent and conductive, which is difficult to obtain because of the optical absorption of the free carriers in the infrared region. In our study, $\mathrm{ZnO}$ (n-type) is use like a window layer to form a hetero-junction with an absorber layer CZTS (p-type). To better understand the impact of $\mathrm{ZnO}$ window layer on the FTO-ZnO-CZTS solar cell's properties, the comparison of the properties of the thin films FTO-CZTS, FTO-ZnO and FTO-ZnO-CZTS layers was made and the results were reported here.

\section{Experimental Details}

\subsection{Precursor Preparation and Depositions Parameters}

In the first step, $\mathrm{ZnO}$ thin films were deposited onto FTO substrat. The sprayed solution is prepared by dissolving zinc chloride $\left(\mathrm{ZnCl}_{2}\right)$ in a mixture of deionized water and ethanol with volume ratio of $7: 3$. The concentration of the precursor solution is set at $0.1 \mathrm{M}$ [22]. Compressed ambient air is used to atomize the solution at the pressure of 2 bars. A volume of $2 \mathrm{~mL}$ solution was sprayed onto the pre-heated glass substrates $\left(2 \times 2 \mathrm{~cm}^{2}\right)$ at $400^{\circ} \mathrm{C}$ [22]. Two types of samples were obtained, using 20 and respectively 40 spraying sequences with $10 \mathrm{~s}$ break between the pulses. The FTO substrats are successively cleaned in an ultrasound bath, using ethanol and distilled water, and were further dried in air. Post-deposition annealing was done for $5 \mathrm{~h}$, in air at $500^{\circ} \mathrm{C}[22]$.

In the second step, the CZTS precursor solution was prepared by dissolving copper chloride $\left(\mathrm{CuCl}_{2}, 1.53432 \mathrm{~g}\right)$, zinc chloride $\left(\mathrm{ZnCl}_{2}, 0.81768 \mathrm{~g}\right)$, tin chloride $\left(\mathrm{SnCl}_{4}\right.$, $1.75285 \mathrm{~g}$ ) in $50 \mathrm{~mL}$ ethanol and stirring for $10 \mathrm{~min}$. All the chemicals used were of analytical grade, as obtained from Sigma Aldrich (99.99\% purity) and were used as received. A volume of $50 \mathrm{~mL}$ of thiourea, with an equal bulk of $3.8048 \mathrm{~g}$ is slowly added drop wise. The concentration of materials was selected as $\mathrm{CuCl}_{2}(0.245 \mathrm{M}), \mathrm{ZnCl}_{2}(0.12 \mathrm{M}), \mathrm{SnCl}_{4}$ $(0.184 \mathrm{M})$ and $\mathrm{CH}_{4} \mathrm{~N}_{2} \mathrm{~S}(1 \mathrm{M})$ in the mass ratio of 1.8:1.2:1:10 [23]. Excess amount of thiourea was required to compensate the loss of sulfur during pyrolysis as sulfur source is volatile at higher temperature [24]. $30 \mathrm{~mL}$ solution was sprayed onto $\mathrm{ZnO}$ film (to obtain FTO-ZnO20-CZTS and FTO-ZnO40-CZTS thin films solar cells) and directly onto the FTO substrat (to obtain FTO-CZTS layer), with the spray rate of $2 \mathrm{~mL} / \mathrm{min}$, and the substrate temperature-set at $300{ }^{\circ} \mathrm{C}$ using a digital temperature controller.

The properties of the deposited films depend on the precursors' solution, spraying rate, substrate temperature, ambient atmosphere, carrier gas, droplet size and the cooling rate after deposition. The film thickness is influenced by the distance between the spraying nozzle and the substrat temperature, the concentration of the precursor and the amount of the sprayed precursor solution [25].

The thin film formation depends on the reactant/solvent evaporation rate and the rate of the process of droplet landing. The ideal deposition condition is considered when the solvent is completely removed at the moment the droplet approaches the substrate where the chemical reaction occurs [26].

The samples are denoted as «FTO-CZTS», «FTO-ZnO20», «TOZnO40», «FTO-ZnO20-CZTS» and «FTO-ZnO40CZTS».

\subsection{Caracterization}

The crystalline structure of the samples was studied by 
XRD using a Bruker D8 Advance Discover Diffractometer with locked coupled continuous scan, using a scintillation counter (12,800 steps; 2s/step) and a radiation with $1.5406 \AA$ wavelength ( $\mathrm{CuK \alpha} 1$, at $40 \mathrm{kV}$ and $20 \mathrm{~mA})$.

The films surface morphology was investigated using a Scanning Electron Microscope (SEM, Hitachi model S-3400 $\mathrm{N}$ type121II) coupled with X-ray energy dispersive spectrometer (EDX) for surface elemental analysis and an Atomic Force Microscope (AFM, NT-MDT model BL222RNTE). The images were taken in semicontact mode with Si-tips (NSG10, force constant $0.15 \mathrm{~N} / \mathrm{m}$, tip radius 10 $\mathrm{nm})$.

Transmittance and reflectance spectra were recorded using a UV-VIS-IR spectro-photometer (Perkin-Elmer Lambda 25 UV/VIS), in the 250-2500 $\mathrm{nm}$ range, with a scan rate of 60 $\mathrm{nm} / \mathrm{min}$ (lamp changes at $326 \mathrm{~nm}$ ).

The band gap energy measurements were obtained using a UV-VIS-IR spectrophotometer (Perkin-Elmer Lambda 25 UV/VIS) in the range from $250 \mathrm{~nm}$ to $2500 \mathrm{~nm}$. The energy value was achieved by extrapolating the $(\alpha . h v)^{2}$ vs $h v$ plot according to Tauc equation.

The I-V (current-voltage) curves in dark and under illumination were obtained using a multichannel potentiostat
(PAR Instruments, model HM8143) with a frequency analyzer; two graphite contacts positioned on the top of the layers and on the substrate's surface and a light source (Oriel, mode 17123) were used. One contact was used for applying voltage (on the FTO substrat) and the second contact was used as a receiver, for recording the current intensity (on the top layer).

\section{Results and Discussions}

\subsection{Structural Properties}

To identify the crystalline phases in the films deposited by SPD, X-ray diffraction (XRD) was carried out. Figure 1. shows XRD pattern of CZTS films deposited on different $\mathrm{ZnO}$ layers. It is evident from the results in Figure 1 that the XRD pattern show three peaks at $2 \theta \sim 28.531^{\circ}, 47.332^{\circ}$ and $56.178^{\circ}$ corresponding to (112), (220) and (312) crystal orientations of CZTS kesterite phase [JCPDS 26-0575], respectively indicating that these films are polycrystalline, for all the film (see stars in red color, Figure 1). The broadening of the XRD peaks (112) indicates the nanocrystalline nature of the films.

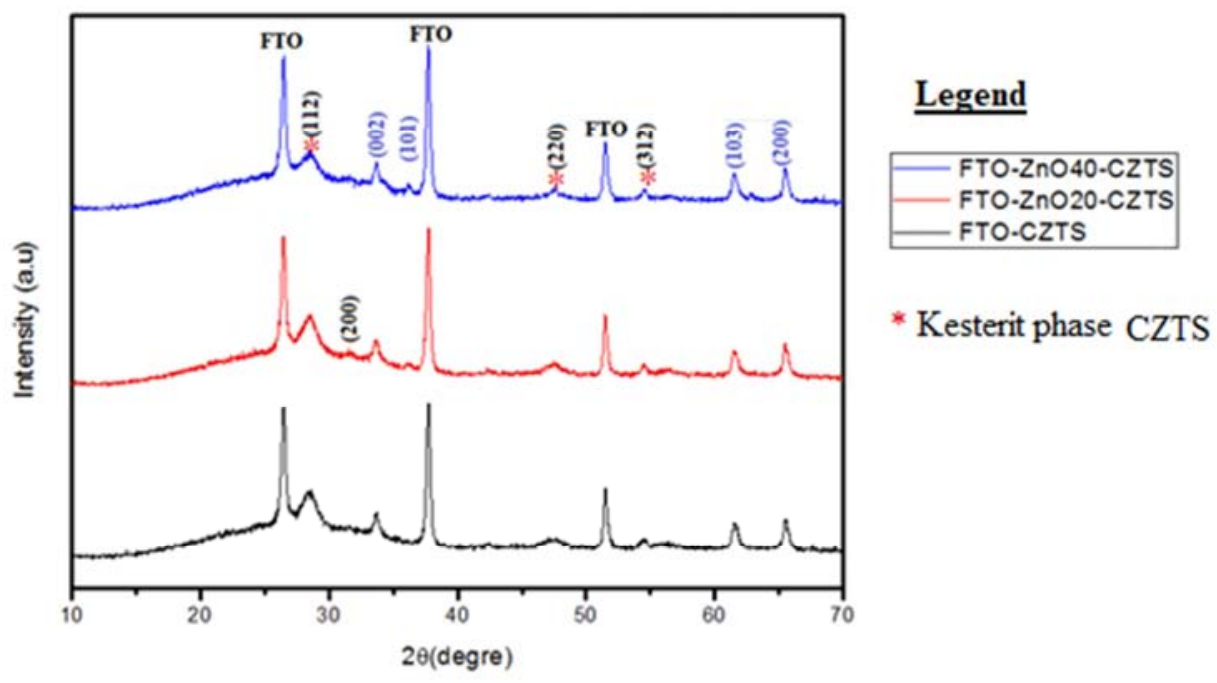

Figure 1. XRD patterns of FTO-CZTS, FTO-ZnO20-CZTS and FTO-ZnO40-CZTS, thin films solar cell.

It is well known that XRD is a limited technique for distinguishing the main CZTS phases from secondary phases as $\mathrm{ZnS}, \mathrm{CuS}$ and $\mathrm{Cu} 2 \mathrm{SnS} 3$ which are formed during the growth process because their peaks are very close to the CZTS ones, as illustrated in Figure 1.

The crystallites size was calculated using Scherrer's equation:

$$
D=\frac{0.9 * \lambda}{\beta^{*} \cos \theta}
$$

Where $\mathrm{D}$ is the crystallite size, $\beta$ is the broading of the diffraction line measured at half of its maximum intensity (rad) $(\mathrm{FWHM})$ and $\lambda$, the X-ray wavelength $\left(1.5418 \mathrm{~A}^{\circ}\right)$. The calculated mean crystallite size is $5.79 \mathrm{~nm}$ for CZTS nanoparticles. It is worth mentioning that the calculated lattice constant of CZTS nanoparticles $(\mathrm{a}=0.5427 \mathrm{~nm}$ and $\mathrm{c}$ $=1.0848 \mathrm{~nm})$, are the same as the value from the standard card $(\mathrm{a}=0.5427 \mathrm{~nm}$ and $\mathrm{c}=1.0848 \mathrm{~nm})$. Similar results were reported by Mou Pal et al [27] with the crystallite sizes ranging from $7.5 \mathrm{~nm}$ to $10.2 \mathrm{~nm}$ by solvothermal method using ethylenediamine as a solvent. The crystallinity estimated for the standard (112) plane showed that crystallinity increases from $31.1 \%$ to $34.8 \%$ respectively for the film FTO-ZnO20-CZTS and FTO-ZnO40-CZTS.

Indeed, crystallinity for films containing only $\mathrm{ZnO}$ is lower than films containing both $\mathrm{ZnO}$ and CZTS. Whereas, for all films, the crystallinity is higher when the amount of $\mathrm{ZnO}$ increases. The FTO-ZnO40-CZTS layer exhibit slightly higher crystallinity as seen in the table 1 . 
Table 1. Cristallinity degree.

\begin{tabular}{ll}
\hline Cell & $\mathbf{C ~ ( \% )}$ \\
\hline FTO-ZnO20 & 31.1 \\
FTO-ZnO40 & 33.7 \\
FTO-CZTS & 33.9 \\
FTO-ZnO20-CZTS & 35.0 \\
FTO-ZnO40-CZTS & 34.8 \\
\hline
\end{tabular}

The sharp peaks at scattering angle $2 \theta$ of $31.71^{\circ}, 34.36^{\circ}$,

\subsection{Morphological Properties}
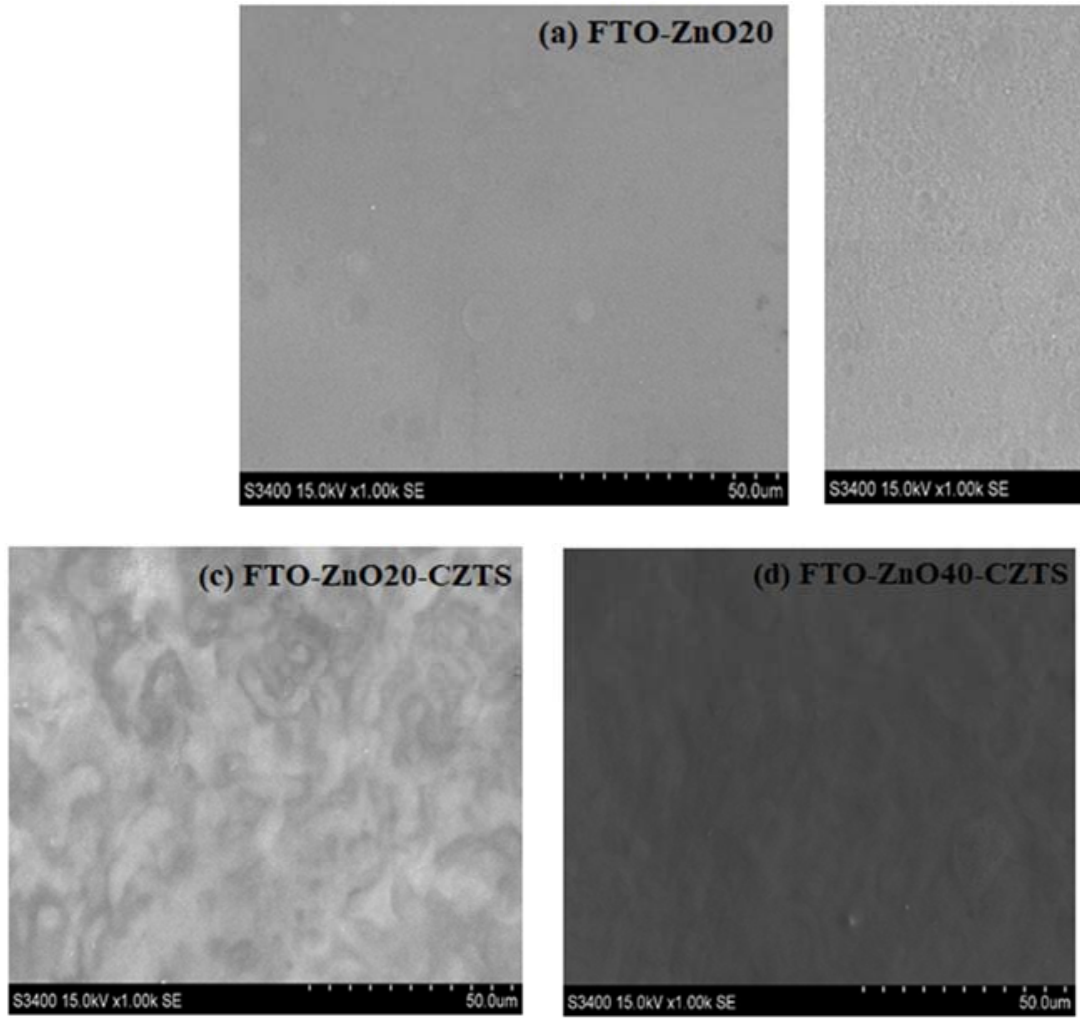

Figure 2. FE-SEM images surface of (a) FTO-ZnO20, (b), FTO-ZnO40, (c) FTO-ZnO20-CZTS, (d) FTO-ZnO40-CZTS and (e) FTO-CZTS thin films after annealing.

The surface morphology of the FTO-ZnO20, FTO-ZnO40, FTO-ZnO20-CZTS, FTO-ZnO40-CZTS, and FTO-CZTS thin films has been studied by SEM and AFM.

The SEM micrographs of films are presented in Figure 2. The surface of FTO-ZnO20 and FTO-ZnO40 films (Figure 2(a) and (b)) is relatively smooth, homogeneous and uniform while that FTO-ZnO20-CZTS, and FTO-CZTS, films respectively (Figure (2c)) and (Figure (2e)) presented some clusters randomly distributed on the films surface, confirming the faster nucleation and the inhomogeneity.

Observation of the SEM images shows that the $\mathrm{ZnO}$-free film (CZTS) lacks uniformity with the presence of greyishcolored heap scattered on the surface of the film. EDX analysis (Table 2) have shown that these heaps consist essentially of sulfur and oxygen. It can be said that the oxygen comes from the substrate (FTO).

Concerning the FTO-ZnO20-CZTS multilayered structure, the CZTS layer weakly and disorderedly covers $\mathrm{ZnO}$, with the formation of whitish-colored clusters throughout the film
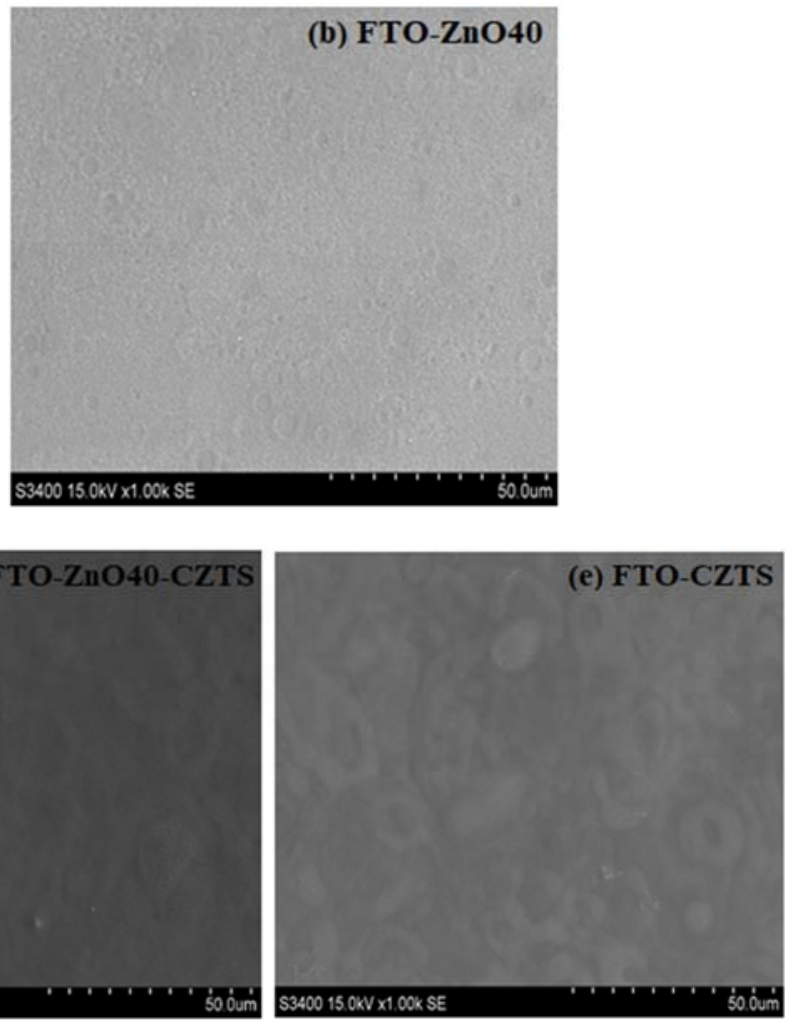

$36.26^{\circ}, 62.83^{\circ}$ and $67.89^{\circ}$ corresponds to the reflection from: (100), (002), (101), (103) and (200) crystal planes, indicating crystalline $\mathrm{ZnO}$ with hexagonal wurzite structure, consistent with the standard JCPDS (Card No.89-0510). No characteristic peaks of impurities could be detected within the precision limit of XRD measurement, which confirms the highly pure and single phase nature of $\mathrm{ZnO}$.

surface. This is due to a small amount of $\mathrm{ZnO}$.

Whereas for the FTO-ZnO40-CZTS thin film, the CZTS layer adheres perfectly to the $\mathrm{ZnO}$ layer. The surface of the film is uniform. It can be said that the amount of $\mathrm{ZnO}$ used here is enough to obtain a better film than the other (FTOZnO20-CZTS).

The AFM micrographs (Figure 3), show that the grains size decreases through films containing only $\mathrm{ZnO}$ (FTO$\mathrm{ZnO} 20$ and FTO-ZnO40), and films containing both $\mathrm{ZnO}$ and CZTS layers (FTO-ZnO20-CZTS and FTO-ZnO40-CZTS), as result of a micro-densification effect. Small crystallites are aggregating to form grains inside the films. The morphology of these grains is different: the film obtained with lower amount of $\mathrm{ZnO}$ (FTO-ZnO20-CZTS) have visibly associated aggregates, well packed and homogeneously distributed on the surface. No local cracks or pin-holes were found by AFM and SEM results. Layers containing only $\mathrm{ZnO}$ showed large grains, while those containing both $\mathrm{ZnO}$ and CZTS showed smaller grains. 
The film FTO-ZnO20 (Figure 3(a)) shows small grains grouped in aggregats. While that of FTO-ZnO40 (Figure 3b) indicates well-ordered larger grains. The grains roughness decreases with increase of $\mathrm{ZnO}$ amount for the FTO-ZnO
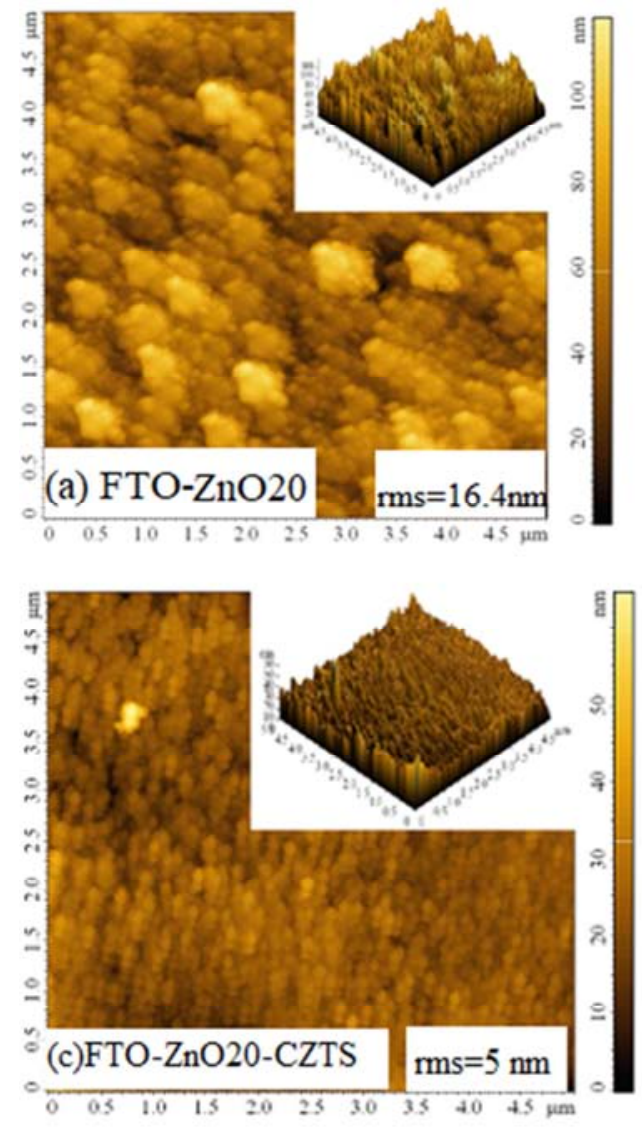

layers, while opposite effect can be outlined for the FTO$\mathrm{ZnO}-\mathrm{CZTS}$, as seen in the table 2, due to a faster nucleation on the $\mathrm{ZnO}$ layer.
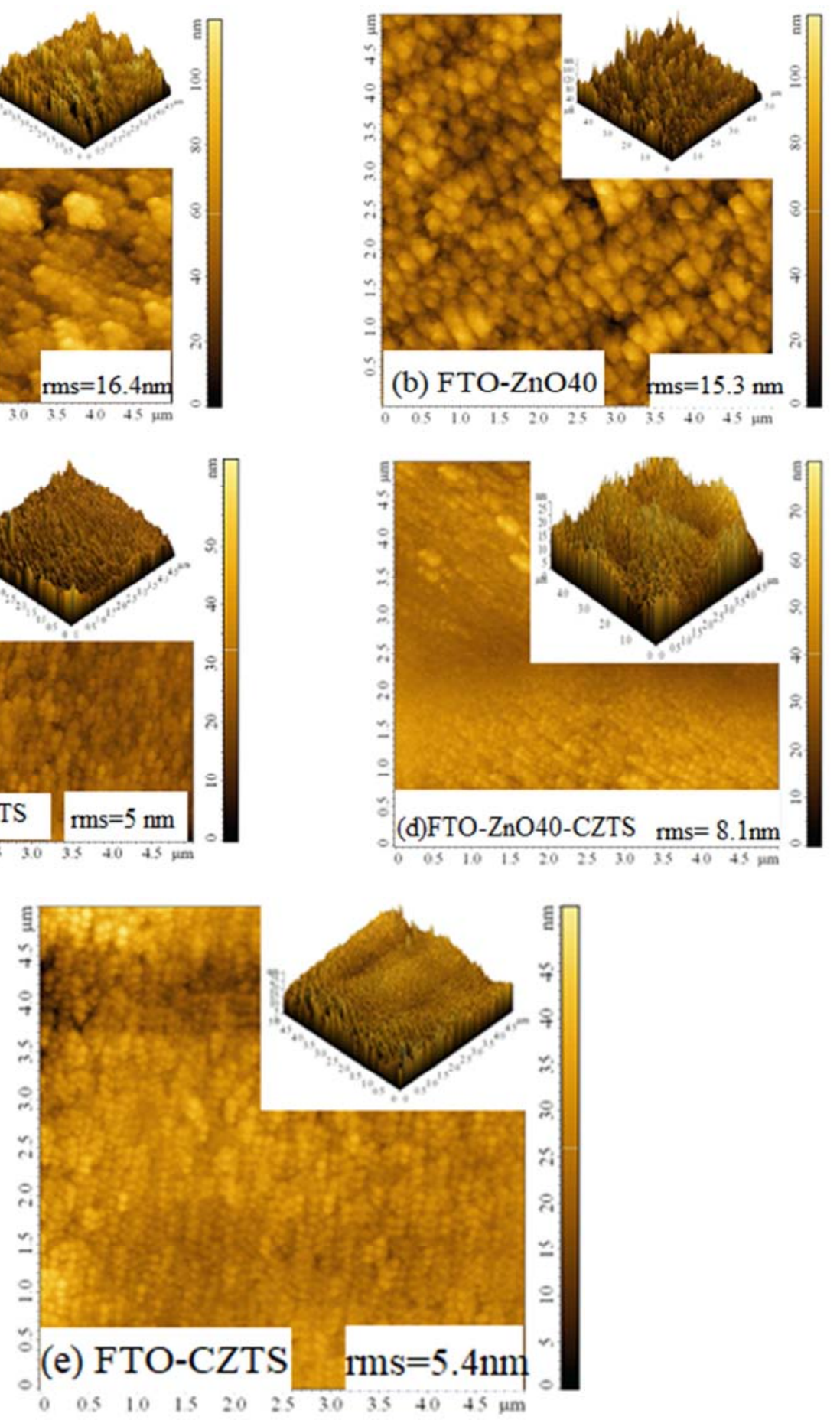

Figure 3. AFM images of thin films (a) FTO-ZnO20, (b) FTO-ZnO40, (c) FTO-ZnO20-CZTS, (d) FTO-ZnO40-CZTS, and (e) FTO-CZTS after annealing.

Table 2. Atomic Percentage of the EDX results.

\begin{tabular}{lllllll}
\hline Thin film & C & O & S & Cu & Zn & Sn \\
\hline FTO-ZnO20-CZTS & 11.99 & 31.245 & 24.735 & 9.36 & 9.10 & 13.57 \\
FTO-ZnO40-CZTS & 13.95 & 26.48 & 22.54 & 9.62 & 12.47 & 14.94 \\
FTO-CZTS & 12.15 & 20.84 & 26.10 & 12.72 & 13.86 & 14.33 \\
\hline
\end{tabular}

Roughness that promotes internal scattering, leads to less transparent layers $[28,29]$, while it is more desirable to have a PV cell that absorbs the maximum of photons from the incident light.

The AFM images (3D) show grains orthogonal to the film 
surface, indicating that the film grows perpendicularly to the substrat as expected.

\subsection{Optical Properties}

The spectra of $\mathrm{ZnO}$ thin films deposited on the FTO substrate are recorded as a function of wavelength in the range of 500-2500 $\mathrm{nm}$ and shown in Figure 4(c). All films are mostly transparent in the visible region and highly absorbent in Near infrared region (NIR). The maximum of transmittance recorded is $85 \%$ for FTO- $\mathrm{ZnO} 20$ and FTO$\mathrm{ZnO} 40$ films in the VIS region. As it can be seen, the transmittance slightly varies with the $\mathrm{ZnO}$ amount.

Concerning the FTO-ZnO-CZTS and FTO-CZTS thin films, the optical transmittance of these films as a function of wavelength in range of 500 to $2500 \mathrm{~nm}$ were recorded and presented in Figure 4 (a). All films are highly absorbent in the visible region. In this area, photons with larger energy than the band gap of the films are sufficient for the excitation of the electrons from the valence band to the conduction band. That is important for a good photocurrent collection. In NIR-region (780-2500 nm), films became mostly transparent through high wavelength. The maximum of transmittance is varied between $65 \%$ and $62.5 \%$. It gradually decreases in the hight wavelength region, up to $5 \%$, indicating an absorbing aspect of the films in this area, what is desirable for a PV cell. This is due to the high absorption coefficient of the CZTS thin film.

The reflectance spectra of the films presented in Figure 4 (b) display that the optical reflectance varies over the range $10-20 \%$ in the VIS range for FTO-ZnO20-CZTS and FTO$\mathrm{ZnO} 40-\mathrm{CZTS}$. The lowest reflectance corresponds to the FTO-CZTS layer due to its high absorption coefficient. The maximum reflectance varies from $55 \%$ to $45 \%$ for a wavelength equal to $2500 \mathrm{~nm}$ The FTO-CZTS film has the lowest reflectance value in the infrared region (IR) region. Therefore the FTO-CZTS film is considered to be suitable for use as an absorbent material for photovoltaic applications (see ref. [2])
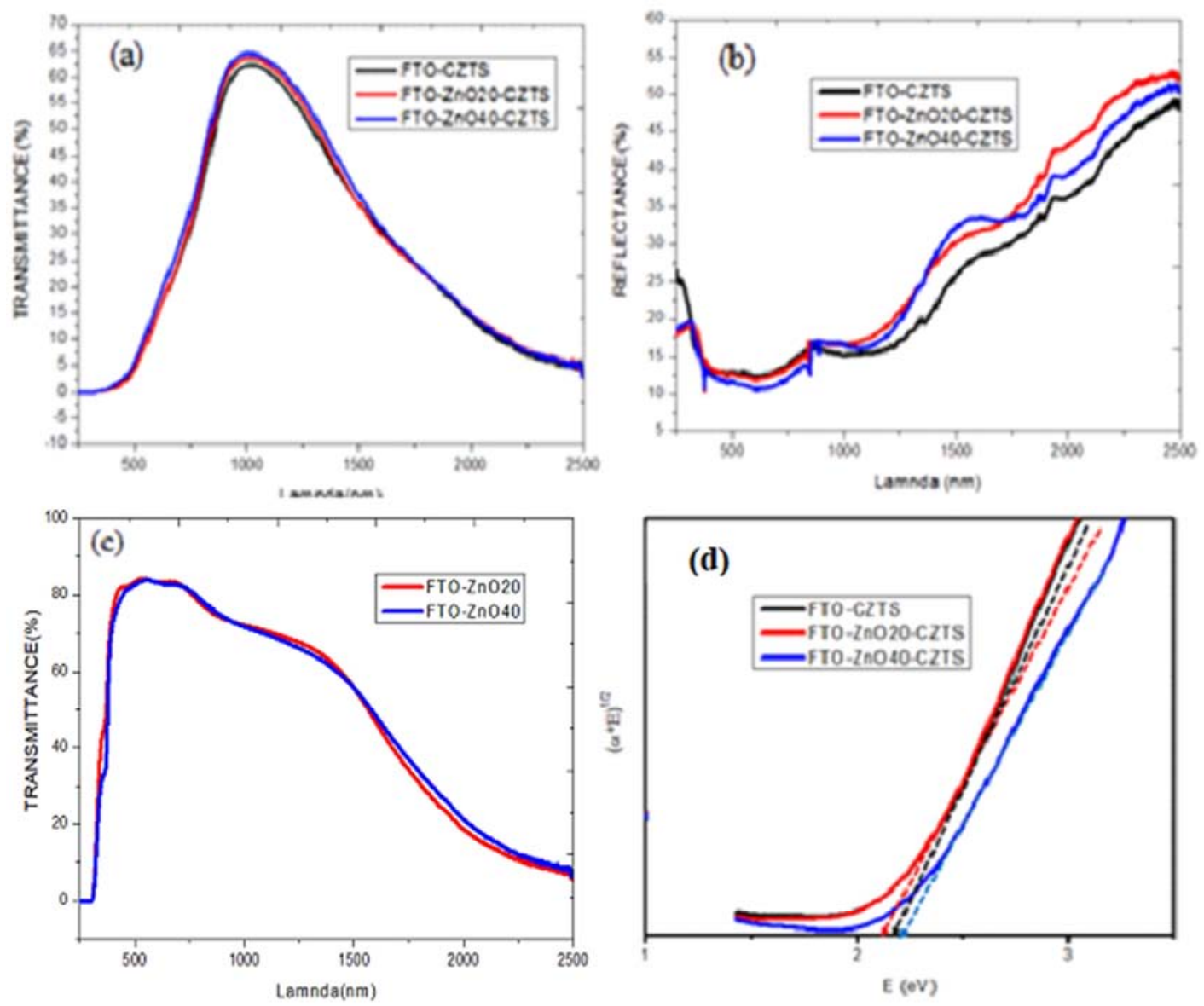

Figure 4. Transmittance (a) and (c), Reflectance (b) and Tauc plot (d) of thin film FTO-CZTS, FTOZnO20-CZTS, FTO-ZnO40-CZTS, FTO-ZnO20 and FTOZnO40 multi-layerd structures.

From transmittance and reflectance measurements, the information on band-gap was found out based on the Tauc plot. The results are presented in Figure 4 (d). It can be seen that no significant change in band gap energies with $\mathrm{ZnO}$ amount. The band gap increases very slightly from $2.1 \mathrm{eV}$ to $2.25 \mathrm{eV}$, because of a slight crystallinity changes of FTO-
ZnO20-CZTS, FTO-ZnO40-CZTS and FTO-CZTS films. The band-gap values in the range of $2.2-2.3 \mathrm{eV}$ are often reported for CZTS compound [30]. The band gap of the material that maximizes absorption of incident light is highly desirable. 


\subsection{Electrical Properties}
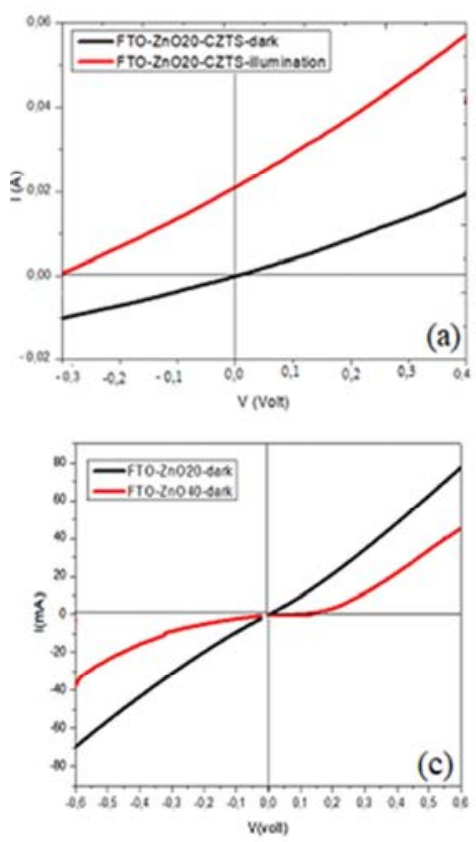
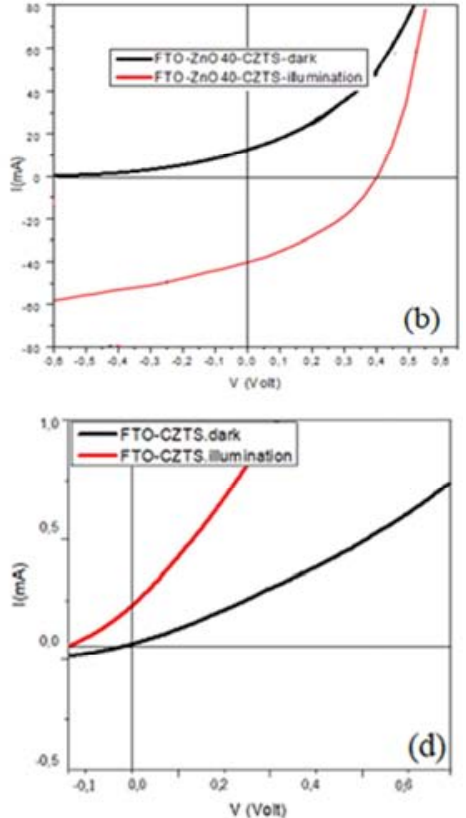

Figure 5. I-V curves of a (a) FTO-ZnO20-CZTS, (b) FTO-ZnO40-CZTS, FTO-ZnO20 and FTO-ZnO40, (d) and FTO-CZTS thin film.

The photo response behavior of ZnO-CZTS, ZnO and CZTS deposited onto FTO substrat was studied in order to evaluate its potential as an active layer for photovoltaic applications. I$\mathrm{V}$ curves of the samples were measured both in the dark and under illumination using a solar simulator (AM $1.5 \mathrm{G}$ irradiation, $100 \mathrm{~mW} . \mathrm{cm}^{-2}$ ). The FTO-ZnO20-CZTS exhibited nearly linear current-voltage curve. The current increases with voltage, which implies the ohmic nature of the contacts. Enhancement in current was observed under illumination for the sample FTO-ZnO20-CZTS, FTO-ZnO40-CZTS and FTOCZTS as shown in Figure 5. The FTO-ZnO40-CZTS film exhibited the conversion efficiency $(\eta)$ of $5.99 \%$ with fill factor $(\mathrm{FF})=18.8 \%$, open circuit voltage $(\mathrm{Voc})=0.54 \mathrm{~V}$, short circuit current density $(\mathrm{Jsc})=59 \mathrm{~mA} . \mathrm{cm}^{2}$. We also noted that highest conductivity, corresponding to a resistive behavior, has been obtained for FTO-ZnO20 thin film in dark. This can be explained, by the fact that the film FTO-ZnO20 in dark has better surface homogeneity and uniformity, as it can be observed in the images of SEM surface. The highest conductivity obtained with FTO-CZTS film can be also due to the increase in electronmobility as a consequence of the grains size enhancement.

\section{Conclusion}

In this paper, the influence of $\mathrm{ZnO}$ amount on the structural, optical, morphological and electrical properties of CZTS thin films has been investigated. The analysis of XRD patterns indicate polycrystalline films, for all the samples. It has been seen that the crystallinity is higher when the amount of $\mathrm{ZnO}$ increases. FE-SEM study shows that more the number of spraying sequences of $\mathrm{ZnO}$ increases, less the layers exhibit aggregates and become more homogeneous as seen in Figure. 2 (b) and Figure 2 (d). The AFM micrographs, confirmed that the grains size increases with increase of $\mathrm{ZnO}$ amount. It has been seen that roughness decrease with increasing the number of spraying sequences of $\mathrm{ZnO}$ for films containing only $\mathrm{ZnO}$ layer. Whereas roughness increase when increasing $\mathrm{ZnO}$ amount in the film that containing both $\mathrm{ZnO}$ and CZTS layers. Furthermore the band gap of the thin film FTO-ZnO-CZTS as seen in the Figure 4 (d) increases very slightly when the amount of $\mathrm{ZnO}$ increases. The optical transmittance spectrum shows that films with only $\mathrm{ZnO}$ layer are mostly transparent in the visible region and high absorbent in IR. While those of films with both $\mathrm{ZnO}$ and CZTS layers are highly absorbent in the visible and IR region. But maximum transmittance of $65 \%$ has been recorded for FTO-ZnO-40-CZTS PV thin film in the NIF region (Near Infrared region). Optical reflectance spectrum shows that the maximum reflectance varies from $55 \%$ to $45 \%$ and the CZTS absorber layer has the lowest reflectance value in the IR region that suitable for use as an absorbent material for photovoltaic applications. We retain that the FTO-ZnO40-CZTS thin film has the best morphological and optical properties, compared to those FTO-ZnO20-CZTS, FTO-CZTS. The FTO-ZnO40-CZTS heterojunction solar cell exhibited the photovoltaic performance with $\eta$ of $5.59 \%$ and FF of $18.8 \%$. This result suggests that $\mathrm{ZnO}$ can be an attractive Cd-free buffer candidate for CZTS based solar cells.

\section{Acknowledgements}

The financial supports of the projects: AUF Eugen Ionesco doctoral scholarship program number 10 and the Romanian government are gratefully acknowledged. 


\section{References}

[1] S. M. Bhosale, M. P. Suryawanshi, J. H. Kimb, A. V. Moholkar, «Influence of copper concentration on sprayed CZTS thin films deposited at high temperature», Ceramics International 41 (2015) 8299-8304.

[2] C. H. Chung, B. Bob, B. Lei, S. H. Li, W. W. Hou, Y. Yang, «Hydrazine solution-processed $\mathrm{Cu}$ In $(\mathrm{Se}, \mathrm{S}) 2$ thin film solar cells: secondary phases and grain structure», Sol. Energy Mater. Sol. Cells113 (2013)148-152.

[3] H. Katagiri, K. Jimbo, W. S. Maw, K. Oishi, M. Yamazaki, H. Araki, A. Takeuchi, «Development of CZTS based thin film solar cells», Thin Solid Films517 (2009)2455-2460.

[4] E. M. Mkawi, K. Ibrahim, M. K. M. Ali, A. S. Mohamed, « Dependence of copper concentration on the properties of $\mathrm{Cu} 2 \mathrm{ZnSnS} 4$ thin films prepared by electrochemical method", Int. J. Electrochem. Sci.8 (2013)359-368.

[5] Shockley W, Queisser HJ. «Detailed Balance Limit of Efficiency of p-n Junction Solar Cells». Jpn J Appl Phys 1961; 32: $510-519$.

[6] H. Katagiri, K. Jimbo, W. S. Maw, K. Oishi, M. Yamazaki, H. Araki, A. Takeuchi, «Development of CZTS-basedthin film solar cells», Thin Solid Films 517 (2009) 2455-2460.

[7] T. Tanaka, D. Kawasaki, M. Nishio, Q. Guo, H. Ogawa, «Fabrication of $\mathrm{Cu} 2 \mathrm{ZnSnS} 4$ thin films by co-evaporation», Phys. Status Solidi C3 (2006)2844-2847.

[8] N. Moritake, Y. Fukui, M. O onuki, K. Tanaka, H. Uchiki, «Preparation of $\mathrm{Cu} 2 \mathrm{ZnSnS4}$ thin film solar cells under nonvacuum condition», Phys. Status Solidi C6 (2009)1233-1236.

[9] Z. Zhou, Y. Wang, D. Xu, Y. Zhang, «Fabrication of $\mathrm{Cu} 2 \mathrm{ZnSnS} 4$ screen printed layers for solar cells», Sol. Energy Mater. Sol. Cells94 (2010)2042-2045.

[10] J. J. Scragg, P. J. Dale, L. M. Peter, G. Zoppi, I. Forbes, « New routes to sustainable photovoltaics: evaluation of $\mathrm{Cu} 2 \mathrm{ZnSnS} 4$ as an alternative absorber material », Phys. Status Solidi B245 (2008)1772-1778.

[11] K. Moriya, J. Watabe, K. Tanaka, H. Uchiki, «Characterization of $\mathrm{Cu} 2 \mathrm{ZnSnS} 4$ thin films prepared by photo-chemical deposition», Phys. Status Solidi C3 (2006) $2848-2852$.

[12] Y. B. Kishore Kumar, G. Suresh Babu, P. UdayBhaskar, V. SundaraRaja, «Preparation and characterization of spraydeposited Cu2ZnSnS4 thin films», Sol. Energy Mater. Sol. Cells93 (2009)1230-1237.

[13] O. Vigil-Galán, M. Espíndola-Rodríguez, M. Courel, X. Fontané, D. Sylla, V. Izquierdo-Roca, A. Fair brother, E. Saucedo, A. Pérez-Rodríguez, «Secondary phases dependence on composition ratio in sprayed $\mathrm{Cu} 2 \mathrm{ZnSnS} 4$ thin films and its impact on the high power conversion efficiency», Sol. Energy Mater. Sol. Cells 117 (2013)246-250.

[14] Maykel Courel, J. A. Andrade-Arvizu, A. Guillén-Cervantes, M. M. Nicolás-Marín, F. A. Pulgarín-Agudelo, O. VigilGalán, «Optimization of physical properties of spraydeposited $\mathrm{Cu} 2 \mathrm{ZnSnS} 4$ thin films for solar cell applications», Materials and Design 114 (2017) 515-520.

[15] B. Shin, O. Gunawan, Y. Zhu, N. A. Bojarczuk, S. J. Chey, S. Guha, «Thin film solar cell with $8.4 \%$ power conversion efficiency using an earth-abundant $\mathrm{Cu} 2 \mathrm{ZnSnS4}$ absorber», Prog. Photovolt. Res. Appl. 21 (2013) 72.

[16] A G Kannan, T E Manjulavalli, J Chandrasekaran, «Influence of solvent on the properties of CZTS nanoparticles», Procedia Engineering 141 (2016) 15-22.

[17] Wujisiguleng Bao, Masaya Ichimura, Band Offsets at the $\mathrm{ZnO} / \mathrm{Cu} 2 \mathrm{ZnSnS} 4$ Interface Based on the First Principles Calculation. Japanese Journal of Applied Physics, 2013, 52, 061203-(1-5).

[18] B. J. Lokhande, M. D. Uplane, «Structural, optical and electrical studies on spray deposited highly oriented $\mathrm{ZnO}$ films», Appl. Surf. Sci.167 (2000) 243-246.

[19] E. Fortunato, P. Barquinha, A. Pimentel, L. Pereira, A. Gonc, alves, A. Marques, R. Martins, Wide-band gap high-mobility $\mathrm{ZnO}$ thin-film transistors produced at room temperature, Appl. Phys. Lett. 85 (2004) 2541-2543.

[20] M. H. Valdés, M. Berruet, A. Goossens, M. Vázquez, Spray deposition of CuInS2 on electrodeposited $\mathrm{ZnO}$ for low-cost solar cells, Surf. Coat. Tech. 204(2010)3995-4000.

[21] Y. Lare, M. Baneto, L. Cattin, M. Morsli, K. Jondo, K. Napo, J. C. Bernède, «Effect of a zinc oxide, at the cathode interface, on the efficiency of inverted organic photovoltaic cells based on theCuPc/C60couple», J. Mater. Sci.: Mater. Electron. 22 (2011) 365-370.

[22] Mazabalo Baneto, Alexandru Enesca, Yendoubé Lare, Koffi Jondo, Kossi Napo, Anca Duta «Effect of precursor concentration on structural, morphological and opto-electric properties of $\mathrm{ZnO}$ thin films prepared by spray pyrolysis», Ceramics International 40 (2014)8397-8404.

[23] Katagiri H, Jimbo K, Tahara M, Araki H, Oishi K. «The influence of the composition ratio on CZTS-based thin film solar cells». In: Materials research society symposium proceedings; 2009. p. M01-4.

[24] Bwamba Jonah A, Alu Noble, Adama Kenneth K., Abdullahi Zakari, Iwok Unwana U., Egba Augustine C., Oberafo Anthony A. «Characterization of CZTS Absorbent Material Prepared by Field-Assisted Spray Pyrolysis», American Journal of Materials Science 2014, 4 (3): 127-132.

[25] Patil, P. S. Versatility of chemical spray pyrolysis technique. «Materials Chemistry and Physics», 1999, 59, 185-198.

[26] Seboui Z, Cuminal Y, Kamoun N. J Energy Renew Sustain 2013; 5: 023113.

[27] Pal Mou, Mathews NR, Gonzalez R, Silva, Mathew X, " Synthesis of $\mathrm{Cu} 2 \mathrm{ZnSnS} 4$ nanocrystals by solvothermal method ». Thin Solid Films, 2013, 535, 78-82.

[28] Q. Wang, Y. Wen, R. Chen, B. Shan, «A hybrid functional study of the electronic and optical properties of tetragonal $\mathrm{PbO}$-type phase of $\mathrm{ZnO}$ under pressure», J. Alloys Compd.586 (2014)611-615.

[29] Y. Li, J. Meng, «Al-doping effects on structure and optical properties of $\mathrm{ZnO}$ nanostructures», Mater. Lett.117 (2014)260-262.

[30] H. Katagiri, K. Saitoh, T. Washio, H. Shinohara, T. Kurumadani, S. Miyajima, «Development of thin film solar cell based on Cu2ZnSnS4 thin films», Sol. Energy Mater. Sol. Cells 65 (2001) 141. 